\title{
Caprylic and Polygalacturonic Acid Combinations for Eradication of Microbial Organisms Embedded in Biofilm
}

\author{
Joel Rosenblatt*, Ruth A. Reitzel, Nylev Vargas-Cruz, Anne-Marie Chaftari, Ray Hachem \\ and Issam Raad
}

Department of Infectious Diseases, Infection Control \& Employee Health, University of Texas MD Anderson Cancer Center,

Houston, TX, United States

OPEN ACCESS

Edited by:

Sara María Soto,

ISGlobal, Spain

Reviewed by:

Dake Xu,

Northeastern University, China

Nuno Pereira Mira,

Instituto de Bioengenharia e

Biociências (IBB), Portugal

${ }^{*}$ Correspondence:

Joel Rosenblat

jsrosenblatt@mdanderson.org

Specialty section:

This article was submitted to

Infectious Diseases,

a section of the journal

Frontiers in Microbiology

Received: 01 August 2017 Accepted: 28 September 2017

Published: 18 October 2017

Citation:

Rosenblatt J, Reitzel RA, Vargas-Cruz N, Chaftari A-M, Hachem $R$ and Raad I (2017) Caprylic and Polygalacturonic Acid Combinations for Eradication of Microbial Organisms Embedded in Biofilm. Front. Microbiol. 8:1999.

doi: 10.3389/fmicb.2017.01999
There is a need for non-antibiotic, antimicrobial compositions with low toxicity capable of broad-spectrum eradication of pathogenic biofilms in food preparation and healthcare settings. In this study we demonstrated complete biofilm eradication within 60 min with synergistic combinations of caprylic and polygalacturonic (PG) acids in an in vitro biofilm eradication model against representative hospital and foodborne infectious pathogen biofilms (methicillin-resistant Staphylococcus aureus, multidrug-resistant Pseudomonas aeruginosa, Candida albicans, Escherichia coli, and Salmonella enteritidis). Antimicrobial synergy against biofilms was demonstrated by quantifying viable organisms remaining in biofilms exposed to caprylic acid alone, PG acid alone, or combinations of the two. The combinations also synergistically inhibited growth of planktonic organisms. Toxicity of the combination was assessed in vitro on L929 fibroblasts incubated with extracts of caprylic and PG acid combinations using the Alamar Blue metabolic activity assay and the Trypan Blue exclusion cell viability assay. The extracts did not produce cytotoxic responses relative to untreated control fibroblasts.

Keywords: caprylic acid, polygalacturonic acid, synergy, biofilm eradication, infection

\section{INTRODUCTION}

Biofilms are three-dimensional dense communities of microbial cells attached to surfaces that are encased in protective biopolymer matrices secreted by the embedded microbes (Costerton et al., 1999; Donlan and Costerton, 2002). Novel disinfecting approaches to rapidly eradicate microbial biofilms that are broad spectrum, economical, and that do not encourage development of resistant organisms remain a significant need in applications including prevention of illnesses from food preparation and storage as well as prevention and treatment of infections in healthcare settings conducive to biofilm formation such as on dermal wounds and implanted medical devices. The Centers for Disease Control and Prevention report that thousands of people get sick annually from consuming contaminated foods resulting in substantial health and economic burdens (National Center for Emerging and Zoonotic Infectious Diseases, and Division of Foodborne, Waterborne, and Environmental Diseases, 2016). Biofilms formed on surfaces of food processing and storage equipment can be very recalcitrant to eradication and can lead to food spoilage and other related economic costs (Van Houdt and Michiels, 2010). Similarly, a significant proportion of human microbial infections have been reported to be associated with biofilms (Miquel et al., 2016). 
Consequently, effective disinfection technologies that eradicate biofilms are needed for prevention and treatment of biofilmbased infections. Infections where biofilms are present can be especially challenging to treat. For example, biofilms can impair healing of wounds in microbially colonized environments, such as the colon, rectum, and urinary tract (Donlan and Costerton, 2002). Biofilms are also frequently present in chronic dermal wounds and contribute to their recalcitrance in healing (James et al., 2008). Furthermore, surgical site infections in the presence of implanted medical devices pose significant healthcare challenges whose complications are magnified when microbial biofilms form on the device surfaces (Magill et al., 2014). Candida species are the most prevalent fungal colonizer of human tissues and produce a significant proportion of medical device-associated fungemias (Nobile and Johnson, 2015).

A variety of approaches for eradicating biofilms in food handling and healthcare settings have been studied. These include synthetic and naturally derived antimicrobial agents as well as energy-based disinfection (Miquel et al., 2016). Traditional disinfectants are harsh chemical agents that can present undesirable mammalian and ecological toxicities (Christen et al., 2017). Traditional antiseptic agents have also demonstrated mammalian toxicities (Kim et al., 2017). Antibiotics, which are frequently naturally derived have been attractive solutions in these settings because of their lower toxicities. However, the wide use of antibiotics has led to development of antibiotic-resistant (including multidrug resistant) organisms (Centers for Disease Control and Prevention, 2013). Biofilms resist penetration of antibiotics in part due to their extracellular matrix polysaccharides which can bind or otherwise restrict diffusion of antibiotics. Cells in the biofilm phenotype can also exhibit altered metabolic and other behaviors rendering them less susceptible to antibiotics (Van Acker et al., 2014). Additionally, the increasing prevalence of antibiotic-resistant biofilms (Centers for Disease Control and Prevention, 2013) impairs the efficacy of antibiotic therapies. Fungal biofilms can be similarly resistant to traditional antifungal therapeutics due to filtration and binding by their extracellular matrices, and can further reduce antifungal susceptibility through efflux pumps and other biofilm phenotype-specific mechanisms that protect them from antifungal-induced oxidative stresses (Van Acker et al., 2014). Due to the adaptation and continued evolution of increased resistance of biofilms to antibiotics and antifungals, there remains a significant need for improved non-antibiotic compositions that can effectively eradicate microbial biofilms without eliciting antibiotic resistance. Agents that are highly active against a narrow spectrum of organisms can select for biofilm formation by organisms they have weaker activity against. Optimal biofilm disinfection technologies therefore would have low cost, present minimal digestive, topical, and parenteral toxicities and would be capable of rapidly eradicating a wide spectrum of pathogenic microbes including Gram-positive, Gram-negative, and fungal pathogens. Plant-derived natural agents have been researched for their ability to provide optimal biofilm disinfection without undesirable accompanying safety or resistance effects.
Caprylic acid (CAP) is a medium chain fatty acid naturally present in coconut oil and mammalian (including human) breast milk (Jensen et al., 1990; Marina et al., 2009). CAP has been a component of some intravenously administered total parenteral nutrition formulations (Wanten and Calder, 2007; Rayyan et al., 2012). Antimicrobial effects have been reported for protonated CAP - most likely due to its ability to interact with the lipophilic portions of microbial cell membranes and disrupt cell membrane integrity (Pohl et al., 2011). The pK of CAP has been reported as approximately 4.8 (CRC, 2004-2005). In the protonated state, since CAP is a small, uncharged lipophilic molecule, its properties promote rapid penetration of biofilm and intercalation into embedded microbial cell membranes.

Polygalacturonic (PG) acid is naturally derived from pectin which is a structural biopolymer (polysaccharide) present in vegetable and fruit cell walls. Naturally derived PG is partially esterified (usually methoxylated), is usually derived from citrus rind or apple pomace, and may contain minor components of other sugar molecules in its backbone (Sriamornsak, 2013). The $\mathrm{pK}$ of PG varies with degree of esterification but ranges from about 3.5 to 4.1 . At $\mathrm{pHs}$ above the $\mathrm{pK}, \mathrm{PG}$ is usually soluble; however, at $\mathrm{pHs}$ below its $\mathrm{pK}$ it can form gels (Sriamornsak, 2013). The molecular weight of naturally derived PG is typically between 50 and $150 \mathrm{KDa}$ (Sriamornsak, 2013). PG is widely used as a pharmaceutical excipient, in hydrocolloid wound dressings (Munarin et al., 2012) and has been shown to promote antimicrobial activity (Espitia et al., 2014). In light of these properties and since PG has also been shown to be effective in emulsifying lipids (Akhtar et al., 2002) here we studied the biocompatibility and potential synergy of the combination of PG and CAP for biofilm eradication in food handling and medical applications.

\section{MATERIALS AND METHODS}

\section{Microbial Strains}

Testing was conducted using highly virulent Gram-positive, Gram-negative, and yeast pathogens representative of common hospital-acquired (HAI) and food-borne infections. Pathogens tested were methicillin-resistant Staphylococcus aureus (MRSA, MDA 120), multi-drug resistant (MDR) Pseudomonas aeruginosa (MDA 118), Candida albicans (MDA 117), Escherichia coli (MDA 123), and Salmonella enteritidis (ATCC 13076). All MDA numbered pathogens tested were clinical isolates cultured from blood of cancer patients with bacteremia stored in the MD Anderson Infectious Disease organism bank. The S. enteritidis strain was an ATCC standard. For testing organisms were grown from glycerol stock trypticase soy agar $+5 \%$ sheep blood (for bacteria) or sabouraud dextrose agar (for yeast). Each organism was inoculated into Muller Hinton Broth (MHB) and diluted to $0.5 \mathrm{McFarland}$. Further dilutions were made as necessary for testing.

\section{Inhibition of Planktonic Organisms}

To assess potential antimicrobial activity of PG and CAP against planktonic organisms, MIC (PG or CAP) and Checkerboard 
$(\mathrm{PG}+\mathrm{CAP})$ assays were conducted. MICs were determined by microbroth dilutions in accordance with Clinical and Laboratory Standards Institute (CLSI) M07 guidelines (CLSI, 2015). Using round bottom 96-well plates, both HAI and food pathogens were exposed to a range of dilutions of PG $(0,0.25,0.5,0.75$, and $1 \%)$, independently and in combinations (checkerboard) with a range of dilutions of CAP $(0,0.01,0.02,0.03,0.04$, and $0.05 \%)$. Sets of experiments were run where the plates were shaken and not shaken during incubation. MIC was determined by visual scoring for growth. The well with the lowest concentration of drug in which no turbidity was observed corresponded with the MIC for the organism tested.

\section{Time to Biofilm Eradication Testing}

A well-established biofilm colonization model (Kuhn et al., 2002) was employed to test eradication of pathogenic biofilms following different durations of exposure to antimicrobial agents. Briefly, $1 \mathrm{~cm}$ silicone disks were placed in 24-well flat bottom cell culture plates and exposed to $1 \mathrm{ml}$ of human plasma overnight at $37^{\circ} \mathrm{C}$. Biofilm was established on silicone disks by inoculating with challenge organism $(1 \mathrm{ml}$ of $5.5 \times 10^{5} \mathrm{CFU} / \mathrm{ml}$ ) and incubating shaking at $37^{\circ} \mathrm{C}$ for $24 \mathrm{~h}$. All culture liquid was then removed and disks were washed shaking for $30 \mathrm{~min}$ in isotonic sterile saline to remove any remaining planktonic organisms. After washing, disks were exposed at $37^{\circ} \mathrm{C}$ for 30 or $60 \mathrm{~min}$ independently to $1 \mathrm{ml}$ of each test. After exposure, any biofilm remaining on the surface of the silicone disks was assessed by disrupting biofilm via sonicating disks in $5 \mathrm{ml}$ isotonic saline for $15 \mathrm{~min}$. The resulting solution was serially diluted and quantitatively cultured onto Trypticase Soy Agar $+5 \%$ sheep blood (bacteria) or Sabouraud Dextrose Agar (yeast). Complete eradication of the biofilm requires a recovery of no viable colonies following treatment. Recovery of fewer viable organisms than from the control indicates partial eradication of the biofilm. Each time point for each organism for each disinfecting solution was tested with six replicates. To ensure eradication was complete (no surviving dormant or persister cells) from biofilms for which no viable colonies were recovered following the exposure to disinfecting solutions, regrowth experiments were conducted by first exposing biofilm-colonized disks to each experimental solution, then rinsing and subsequently transferring the disks to fresh broth and re-incubating for an additional $24 \mathrm{~h}$. Following the $24 \mathrm{~h}$ regrowth interval, disks were then sonicated and cultured as indicated above to determine whether any organisms remaining embedded in the biofilm were still viable.

Antimicrobial solutions were prepared containing 1\% PG (Sigma-Aldrich, St. Louis, MO, United States, $<15 \%$ esterified) + either 0.1 or $0.4 \%$ CAP (Sigma-Aldrich, St. Louis, MO, United States). 0.1\% CAP was selected because it was near the reported aqueous solubility limit for CAP (Hoerr et al., 1946). The emulsifying capacity of pectins for lipids has been reported near $40 \%$ or more (Liang et al., 2015), hence 1\% PG and $0.4 \%$ CAP ( $40 \%$ of the PG concentration) were selected for testing since $0.4 \%$ CAP was well above the solubility limit of CAP and therefore presented the potential for increasing the effective concentration of CAP presented to microbes. MHB, 1\% PG, $0.1 \%$ CAP, and $0.4 \%$ CAP were included as control solutions. For consistency, the $\mathrm{pH}$ of all test solutions, including $\mathrm{MBH}$ control, was maintained at $\mathrm{pH} 4.25$ with sodium hydroxide or hydrochloric acid as necessary.

\section{Mammalian Cytotoxicity Testing}

Mammalian cytotoxicity testing was conducting following the indirect method of de Gomes et al. (2011) where L929 mouse fibroblasts are exposed to extracts of challenge compositions over a concentration range from 2 to $0.5 \%$ for $24 \mathrm{~h}$ (de Gomes et al., 2011). After exposure, viability was tested using the Alamar Blue Assay (O'Brien et al., 2000) and the Trypan Blue Exclusion staining assay (Strober, 2015). L929 fibroblasts (ATCC \#CCL-1; ATCC, Manassas, VA, United States) were grown in Dulbecco's modified Eagle's medium (DMEM; Corning Cell Grow, Manassas, VA, United States) supplemented with $10 \%$ heat-inactivated fetal bovine serum (FBS; Sigma-Aldrich, St. Louis, MO, United States) in $5 \% \mathrm{CO}_{2}(\mathrm{v} / \mathrm{v})$ at $37^{\circ} \mathrm{C}$. Seeding density of fibroblasts was $4.5 \times 10^{3}$ cells/well in 96well flat bottomed culture plates for Alamar Blue assay and $2.8 \times 10^{5}$ cells in $25 \mathrm{~cm}^{2}$ culture flasks for live dead staining. Following the indirect exposure method of de Gomes et al. (2011), when growth reached approximately $60 \%$ confluence cells were exposed to a 2, 1, and $0.5 \%$ extracts of $1 \%$ PG $+0.4 \%$ CAP in DMEM + 10\% FBS for $24 \mathrm{~h}$. Control (untreated) cells were incubated in DMEM $+10 \%$ FBS without added disinfecting agents. After the exposures, cell viability and toxicity were assessed. All experiments were performed in triplicate.

The Alamar Blue assay (Life Technologies, Corp., Carlsbad, CA, United States) was used to assess the sensitivity of metabolic activity of fibroblasts following exposure to PG + CAP. This assay measures the overall metabolic activity based on production of highly fluorescent resorufin by reducing resazurin in response to enzyme activity in cells (O'Brien et al., 2000). Cytotoxic compounds cause fibroblasts to lose their ability to metabolically reduce resazurin to resorufin thus do not produce the fluorescent signal. After $24 \mathrm{~h}$ exposure to PG + CAP solutions, medium was replaced with $100 \mu \mathrm{l}$ of Hank's Balanced Salt Solution (HBSS; Corning Cell Grow, Manassas, VA, United States) $+10 \%$ Alamar Blue reagent and incubated for $4 \mathrm{~h}$ in $5 \% \mathrm{CO}_{2}(\mathrm{v} / \mathrm{v})$ at $37^{\circ} \mathrm{C}$. Absorbance was determined at $570 \mathrm{~nm}$ using a microplate reader spectrophotometer. Cell viability (absorbance) was compared between treated and untreated control cells. Control (untreated) cells were incubated in DMEM $+10 \%$ FBS and their metabolic activity was assessed by the Alamar Blue assay described above. All experiments were performed in triplicate. Results are expressed as a percentage of fluorescent signal normalized to untreated controls.

The Trypan Blue exclusion test was used as a cell staining test to determine the number of viable cells present in cell suspension (Strober, 2015). Live cells with intact membranes have the ability to exclude the dye Trypan Blue, whereas dead cells do not. Therefore, viable cells had a clear cytoplasm whereas dead cells were stained with blue cytoplasm (Strober, 2015). After $24 \mathrm{~h}$ 
exposure to PG + CAP, cells were washed with HBSS to remove any anti-trypsin serum proteins and harvested from the culture flask with $0.05 \%$ trypsin EDTA (Corning Cell Grow, Manassas, VA, United States). Once detached, DMEM $+10 \%$ FBS was added and cells were pelleted at $200 \times g$ for $7 \mathrm{~min}$. Supernatant was decanted and cells were resuspended in $2 \mathrm{ml}$ of HBSS. Ten microliter of aliquots of cell suspension was stained with $10 \mu \mathrm{l}$ 0.4\% Trypan Blue (Sigma-Aldrich, St. Louis, MO, United States). Live and dead cells were counted with a hemacytometer. Control, untreated cells were incubated in DMEM + 10\% FBS and viability was assessed using the Trypan Blue exclusion test described above. All experiments were performed in triplicate. Results were expressed as percent viable cells in suspension.

\section{Statistical Testing}

The Kruskal-Wallis test was used to determine any significant differences in the medians in any of the solutions tested. Subsequently pairwise comparisons were conducted using the Mann-Whitney $U$-test to compare performance of $1 \% \mathrm{PG}+$ $0.4 \%$ CAP, individual components, and $\mathrm{MHB}$ control at the either 30 or 60 min time points. All comparisons were two-sided with an alpha level of 0.5 . A $p$-value less than $0.05(p<0.05)$ was considered to be statistically significant.

\section{RESULTS}

\section{Inhibition of Planktonic Organisms}

All MICs for individual components of CAP and PG as well as combinations are presented in Table 1. No differences were seen when the plates were shaken or not shaken. For MRSA, inhibition occurred at PG concentrations above $0.5 \%$ (its MIC). Growth of MRSA was completely inhibited at $0.03 \%$ CAP (its MIC). At $0.01 \%$ CAP, $0.25 \%$ PG in combination was able to inhibit MRSA growth. For $P$. aeruginosa, inhibition occurred at PG concentrations above $0.75 \%$. Concentrations of CAP at $0.05 \%$ or less did not inhibit $P$. aeruginosa. At $0.05 \%$ CAP, a PG concentration of $0.25 \%$ in combination was able to inhibit $P$. aeruginosa growth. $C A$ was not inhibited at $\mathrm{PG}$ concentrations of $1 \%$ or less nor at CAP concentrations of $0.05 \%$ or less. Nevertheless, at $0.01 \%$ CAP a PG concentration of $0.25 \%$ in combination was able to inhibit growth of C. ablicans. For E. coli, the MIC of PG was $1 \%$. Concentrations of $0.05 \%$ or less CAP did not inhibit growth of $E$. coli. At a CAP concentration of $0.02 \%, 0.75 \%$ PG in combination was able to inhibit growth of E. coli and similarly at a CAP concentration of $0.04 \%$, $0.5 \%$ PG in combination was able to inhibit growth of E. coli. $S$. enteritidis was not inhibited by PG at concentrations of $1 \%$ or less nor was its growth inhibited at CAP concentrations of $0.05 \%$ or less. At $0.01 \%$ CAP, $0.75 \%$ PG in combination was able to inhibit growth of $S$. enteritidis and similarly at $0.02 \%$ CAP, $0.5 \%$ PG in combination was able to inhibit growth of S. enteritidis.

\section{Time to Biofilm Eradication}

Time to biofilm eradication testing results for broth control, $1 \%$ PG, $0.1 \%$ CAP, $0.4 \%$ CAP, and for the combinations $1 \%$
TABLE 1 | Minimum inhibitory concentration (MIC) of caprylic acid, polygalacturonic acid, and combinations.

\begin{tabular}{lccc}
\hline & MIC PG alone & MIC CAP alone & MIC PG + CAP \\
\hline MRSA & $0.5 \%$ & $0.03 \%$ & $0.25 / 0.01 \%$ \\
Ps. aerguinosa & $0.75 \%$ & $>0.05 \%$ & $0.25 / 0.05 \%$ \\
C. albicans & $>1 \%$ & $>0.05 \%$ & $0.25 / 0.01 \%$ \\
E. coli & $1 \%$ & $>0.05 \%$ & $0.75 / 0.02 \%$ \\
& & & $0.5 / 0.04 \%$ \\
S. enteridits & $>1 \%$ & $>0.05 \%$ & $0.75 / 0.01 \%$ \\
& & & $0.5 / 0.02 \%$
\end{tabular}

MICs were determined by microbroth dilutions in accordance with CSLI (Clinical and Laboratory Standards Institute) M07 guidelines. Pathogens were exposed to a range of dilutions of $P G(0,0.25,0.5,0.75$, and $1 \%)$, independently and in combinations (checkerboard) with a range of dilutions of $\operatorname{CAP}(0,0.01,0.02,0.03$, 0.04 , and $0.05 \%$ ). MIC was determined by visual scoring for growth (">" denotes greater than).

$\mathrm{PG}+0.1 \% \mathrm{CAP}$ and $1 \% \mathrm{PG}+0.4 \% \mathrm{CAP}$ are presented in Figures 1A-E for MRSA, P. aeruginosa, C. albicans, E. coli, and $S$. enteritidis, respectively, section "Results" presented are the median colony forming units per silicone disk with range bars for the six replicates. Efficacy of $1 \%$ PG $+0.4 \%$ CAP in eradicating biofilm was statistically significant for MRSA (60 $\mathrm{min}), P$. aeruginosa (30 $\mathrm{min})$, and $C$. albicans $(60 \mathrm{~min})$ relative to $\mathrm{MHB}$ control ( $p=0.002$ for all organisms) and $1 \%$ PG alone ( $p=0.002, p=0.015$, and $p=0.002$, respectively). Compared to $0.4 \%$ CAP alone, $1 \%$ PG $+0.4 \%$ CAP displayed significant reductions against $P$. aeruginosa at $30 \mathrm{~min}(p=0.041)$ and $C$. albicans at $60 \mathrm{~min}(p=0.015)$. For MRSA $1 \%$ PG $+0.4 \%$ CAP completely eradicated biofilm (no viable colonies recovered) while $0.4 \% \mathrm{CAP}$ alone had a median growth of $6.25 \times 10^{2} \mathrm{CFU}$ per disk, however, this difference did not attain statistical significance $(p=0.18)$. One percent of $\mathrm{PG}+0.4 \%$ CAP completely eradicated $E$. coli within $30 \mathrm{~min}$ (no viable colonies recovered) while $0.4 \%$ CAP had a median growth of $3.55 \times 10^{3} \mathrm{CFU}$ per disk; however, this difference also did not attain statistical significance $(p=0.2)$. Both CAP alone and the combination of PG + CAP was highly effective in eradication $S$. enteritidis biofilm within $30 \mathrm{~min}$ of exposure. There was no regrowth for any solution for which no viable colonies were recovered in the biofilm eradication assay. This verified that eradication was indeed complete when no viable colonies were recovered in the biofilm eradication assay.

\section{Cytotoxicity Testing}

Figure 2 presents results of the Alamar Blue cytotoxicity assay for control fibroblasts as well as fibroblasts exposed to 2, 1, and $0.5 \%$ extracts of $1 \% \mathrm{PG}+0.4 \%$ CAP. Results are expressed as percentage fluorescence signal relative to the control. There were no significant differences $(p>0.31)$ in metabolic activity between any of the groups compared to untreated control cells. Based on counting 1.5-2.0 $\times 10^{6}$ cells in each of three replicates, the control in the Trypan Blue cell exclusion assay yielded an average $96.9 \%$ viable cells (clear cells). The $2 \%$ extract of $1 \%$ PG + $0.4 \%$ CAP yielded an average $97.6 \%$ viable cells (Table 2 ). There was no significant difference in cell viability between the groups $(p=0.41)$. 

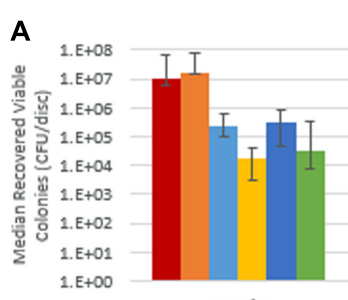

$30 \mathrm{~min}$

MRSA (MDA 120)

C
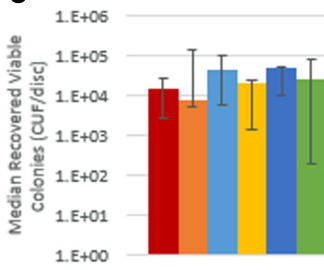

$30 \mathrm{~min}$

C. albicans (MDA 117)

E

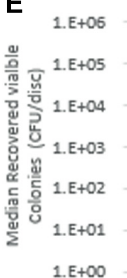

1. $E+\infty$

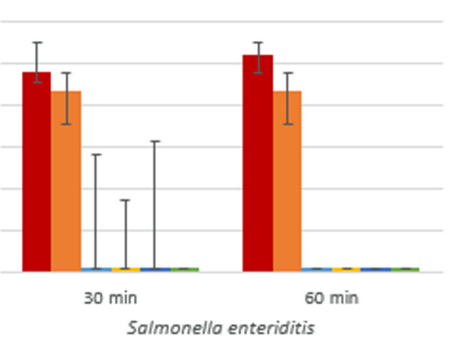

- MHBControl $=1 \% P G=0.1 \%$ CAP $=0.4 \%$ CAP $=1 \% P G+0.1 \%$ CAP $\equiv 1 \% P G+0.4 \%$ CAP

- MHB control

119PG

- $0.1 \%$ CAP

$=0.4 \% \mathrm{CAP}$

- $1 \% \mathrm{PG}+0.1 \%$ CAP

a $1 \% \mathrm{PG}+0.4 \%$ CAP
$B_{1 . E+06}$

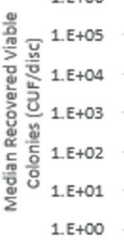
1. $E+\infty$

D

1. E+06

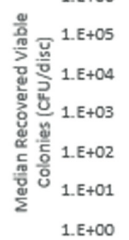
1. $E+\infty 0$

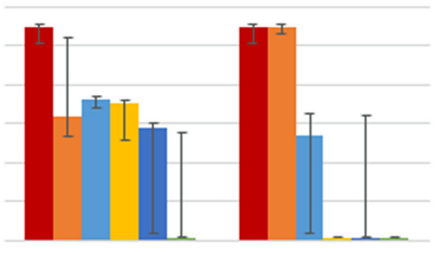

$30 \mathrm{~min}$

$60 \mathrm{~min}$

Escherchia coli (MDA 123)

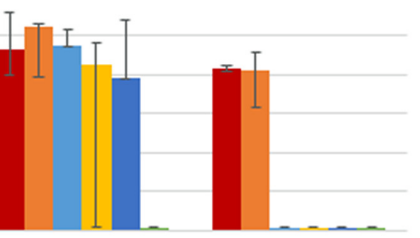

$60 \mathrm{~min}$

P. aeruginosa (MDA 118)

$$
\text { ) }
$$
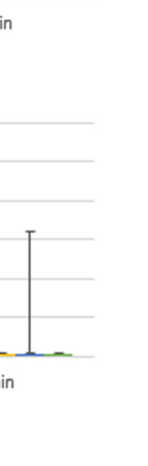

FIGURE 1 | Synergistic eradication of biofilms from representative infectious pathogens - MRSA (A), P. aeruginosa (B), C. albicans (C), E. coli (D), and S. enteritidis (E) by polygalacturonic acid (PG) and caprylic acid (CAP) combinations. All figures are presented as median recovered viable colonies with range bars.

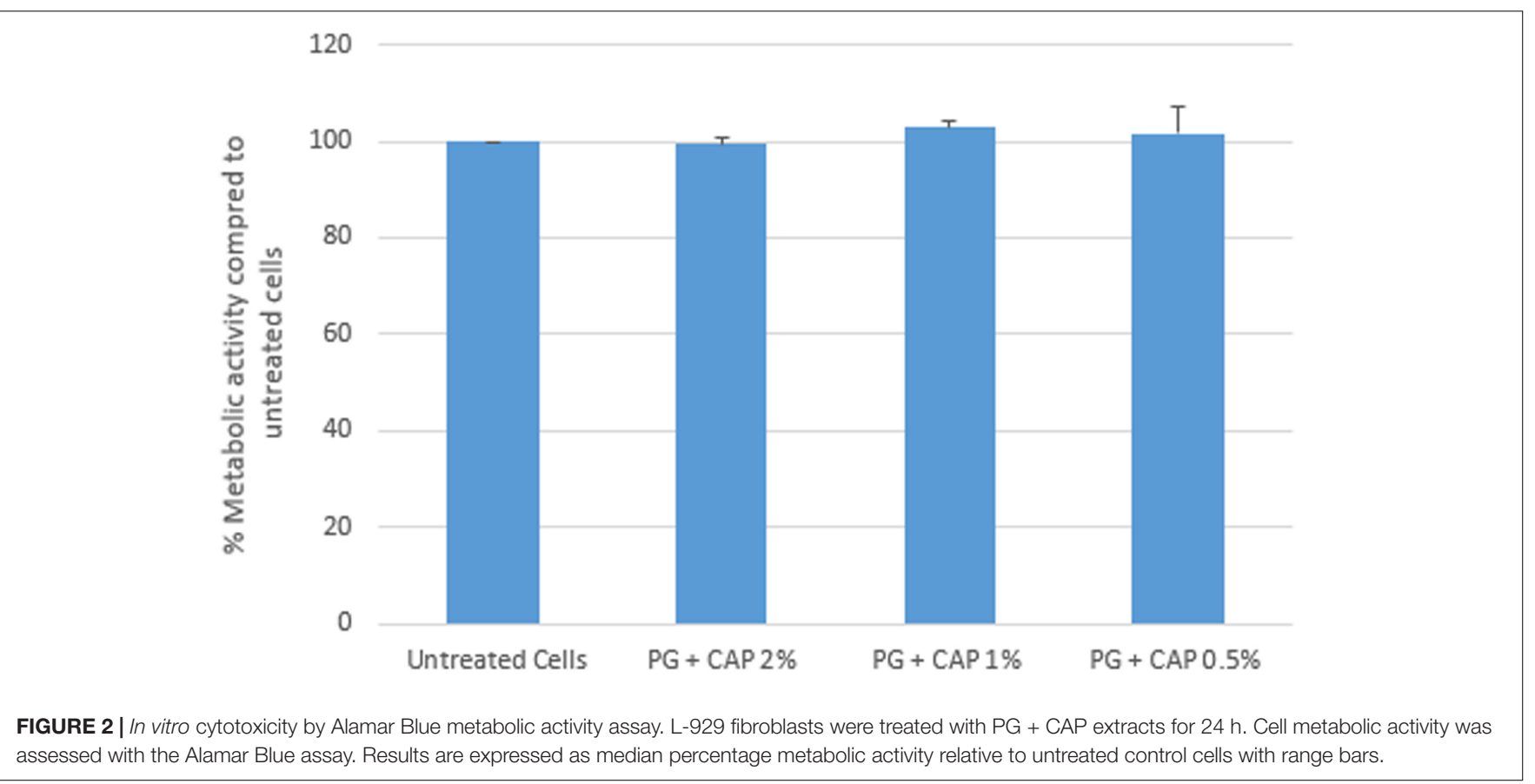


TABLE 2 | In vitro cytotoxicity assessment of cell viability by Trypan Blue staining: L-929 Fibroblasts were treated with PG + CAP extracts for $24 \mathrm{~h}$.

\begin{tabular}{lcc}
\hline & $\begin{array}{l}\text { Untreated L929 } \\
\text { Cells (cells } / \mathbf{m L})\end{array}$ & $\begin{array}{c}\text { L929 Fibroblasts } \\
\text { treated with 1\% } \\
\mathbf{P G}+\mathbf{0 . 4 \%} \\
\mathbf{C A P}(\mathbf{c e l l s} / \mathbf{m L})\end{array}$ \\
\hline $\begin{array}{l}\text { Mean Live Cells } \pm \text { standard } \\
\text { deviation }\end{array}$ & $1.84 \pm 0.18 \times 10^{6}$ & $1.57 \pm 0.11 \times 10^{6}$ \\
$\begin{array}{l}\text { Mean Dead Cells } \pm \text { standard } \\
\text { deviation }\end{array}$ & $6.00 \pm 2.12 \times 10^{4}$ & $3.83 \pm 0.60 \times 10^{4}$ \\
$\%$ Viable & $96.89 \%$ & $97.62 \%$ \\
\hline
\end{tabular}

Cell viability was assessed with the Trypan Blue exclusion assay. Results are expressed as percentage viable cells relative to untreated control cells.

\section{DISCUSSION}

As shown in Figure 1, protonated CAP at both 0.1 and $0.4 \%$ concentrations demonstrated reduction in viable MRSA and C. albicans organisms in biofilms after $60 \mathrm{~min}$ incubation, however, complete eradication was only seen against $P$. aeruginosa biofilms within $60 \mathrm{~min}$. Previously, CAP was reported to have antimicrobial activity against planktonic Grampositive and Gram-negative organisms including $P$. aeruginosa and MRSA (Kim and Rhee, 2016) but its effectiveness against biofilm was not assessed. Other than for E. coli (Figure 2), there was no significant impact of increasing the CAP concentration from 0.1 to $0.4 \%$ on biofilm eradication when no PG was present. This is consistent with the reported aqueous solubility limit of CAP of approximately $0.07 \%$ (Hoerr et al., 1946), hence once the CAP concentration approached saturation there was little impact on biofilm eradication from having insoluble CAP present. Although PG alone demonstrated inhibitory activity against planktonic $P$. aeruginosa, MRSA, and E. coli there was no appreciable antimicrobial effect against $P$. aeruginosa, E. coli, and MRSA biofilms. This may have been a result of the molecular size and electrostatic charge of PG whose access to embedded microbial cells within biofilms may have been reduced by interactions with extracellular biofilm matrix polysaccharides. PG alone had limited effectiveness against planktonic C. albicans and S. enteritidis or biofilms of C. albicans and S. enteritidis.

In this study, a synergistic reduction in the time to biofilm eradication was seen with the $1 \% \mathrm{PG}+0.4 \%$ CAP combination against MRSA (at $60 \mathrm{~min}$ ), P. aeruginosa (at $30 \mathrm{~min}$ ), E. coli (at $30 \mathrm{~min}$ ), and C. albicans (at $60 \mathrm{~min}$ ). One percent PG + $0.1 \%$ CAP demonstrated synergistic reduction in time to biofilm eradication against E. coli (60 min). The PG + CAP combinations were also highly effective against $S$. enteritidis biofilms, but CAP alone was also highly effective against $S$. enteritidis biofilms at the exposures tested ( 30 and $60 \mathrm{~min}$ ). Since the broth for the controls was adjusted to the same $\mathrm{pH}(4.25)$ as the antimicrobial solutions, the antimicrobial effects were not due to solution acidity. It is possible that synergy of the PG + CAP combinations against $S$. enteritidis biofilms was present at exposure times shorter than those tested in this study. A hypothesis for the enhanced synergy seen with $0.4 \%$ CAP in combination with
PG may be that the apparent solubility of CAP is enhanced in the PG + CAP combination and the bioavailability of both CAP and PG within biofilms is significantly increased when combined. At $0.4 \%$ concentration, CAP alone (without PG) showed visible signs of phase separation or immiscibility and was well above the reported aqueous CAP solubility limit of $0.07-0.08 \%$ at $25^{\circ} \mathrm{C}$ (Hoerr et al., 1946). This is also consistent with the minor differences in antimicrobial activity seen between CAP alone at 0.1 and $0.4 \%$ in Figure 1 where soluble CAP might have saturated. Observations of $1 \%$ PG $+0.4 \%$ CAP through an optical microscope showed the presence of finely emulsified oil droplets consistent with prior reports of the lipid emulsifying properties of PG (Akhtar et al., 2002). The planktonic data for PG and CAP combinations support an additional hypothesis for dual mechanisms of antimicrobial synergy for the combination beyond increasing the bioavailability of CAP and PG within the biofilms. While protonated CAP is known to directly disrupt cell membranes (Pohl et al., 2011), PG is capable of binding or precipitating important metal ions and molecules with cationic residues (Espitia et al., 2014) such as proteins and peptides. These activities inhibit microbial proliferation and survival, particularly when cell membranes are simultaneously stressed and made more porous by the action of protonated CAP.

Caprylic acid is a naturally occurring fatty acid. In the ionized (deprotonated) state (neutral $\mathrm{pH}$ ), caprylate ion is a nutrient with a well-established metabolic profile in mammals (Hirabara et al., 2006). CAP can become ionized when the $\mathrm{pH}$ rises above 4.8 (CRC, 2004-2005), hence in most physiological environments CAP will rapidly ionize to a benign nutrient. In a topical formulation, protonated CAP which retains antimicrobial activity can be present by maintaining a $\mathrm{pH}$ below 4.8 . This is within the natural $\mathrm{pH}$ range seen in human skin (Lambers et al., 2006). Additional epithelial environments such as the vaginal canal (Lang, 1955) and digestive tract (Evans et al., 1988) also naturally maintain acidic pHs. Dermal wounds have been reported to exhibit improved healing at acidic $\mathrm{pHs}$ (Schreml et al., 2010). Honey, which has a pH of 3.2-4.2 (Pieper, 2009) has been directly applied to and used to treat wounds. Some clinical trials have reported an enhancement of healing with topical application of honey and the safe topical use of acidic, medical grade honey in wounds has been consistently reported (Lee et al., 2011). Wound healing has also been reported to be aided by the presence of CAP in wound beds (Pieper and Caliri, 2003; Srivastava and Durgaprasad, 2008). Due to its pK, PG naturally maintains a $\mathrm{pH}$ in the same range as honey. PG has been widely used in hydrocolloid wound dressings with reported benefits of maintaining a moist, acidic environment, and providing a bacterial barrier and is in several commercial wound healing sheet and paste products (Munarin et al., 2012).

\section{CONCLUSION}

We found that PG and CAP combinations are capable of rapidly eradicating representative Gram-positive, Gram-negative, and 
fungal biofilms within $60 \mathrm{~min}$. We further found that the combination does not induce a cytotoxic response in mammalian fibroblasts. This in addition to the prior history of safe use of the individual components in wound healing and nutrition applications suggests that the combination should be suitable for topical and food disinfection uses. The synergistic, nonantibiotic, antimicrobial combination requires further in vivo testing to substantiate both its safety and efficacy.

\section{REFERENCES}

Akhtar, M., Dickinson, E., Mazoyer, J., and Langendorff, V. (2002). Emulsion stabilizing properties of depolymerized pectin. Food Hydrocoll. 16, 249-256. doi: 10.1016/S0268-005X(01)00095-9

Centers for Disease Control and Prevention (2013). Antibiotic Resistance Threats in the United States, 2013. Atlanta, GA: Centers for Disease Control and Prevention.

Christen, V., Faltermann, S., Brun, N. R., Kunz, P. Y., and Fent, K. (2017). Cytotoxicity and molecular effects of biocidal disinfectants (quaternary ammonia, glutaraldehyde, poly(hexamethylene biguanide) hydrochloride PHMB) and their mixtures in vitro and in zebrafish eleuthero-embryos. Sci. Total Environ. 586, 1204-1218. doi: 10.1016/j.scitotenv.2017. 02.114

CLSI (2015). M07-A10 - Methods for Dilution Antimicrobial Susceptibility Tested for Bacteria that Grow Aerobically; Approved Standard, 10th Edn. Wayne, PA: CLSI.

Costerton, J. W., Stewart, P. S., and Greenberg, E. P. (1999). Bacterial biofilms: a common cause of persistent infections. Science 284, 1318-1322. doi: 10.1126/ science. 284.5418 .1318

CRC (2004-2005). Handbook of Chemistry and Physics. New York, NY: CRC Press.

de Gomes, P. S., Figueiral, M. H., Fernandes, M. H., and Scully, C. (2011). Cytotoxicity of denture adhesives. Clin. Oral Investig. 15, 885-893. doi: 10.1007/ s00784-010-0464-0

Donlan, R. M., and Costerton, J. W. (2002). Biofilms: survival mechanisms of clinically relevant microorganisms. Clin. Microbiol. Rev. 15, 167-193. doi: 10. 1128/CMR.15.2.167-193.2002

Espitia, P., Du, W., Avena-Bustillos, R., Soares, N., and McHugh, T. (2014). Edible films from pectin: physical-mechanical and antimicrobial properties - A review. Food Hydrocoll. 35, 287-296. doi: 10.1016/j.foodhyd.2013.06.005

Evans, D. F., Pye, G., Bramley, R., Clark, A. G., Dyson, T. J., and Hardcastle, J. D. (1988). Measurement of gastrointestinal $\mathrm{pH}$ profiles in normal ambulant human subjects. Gut 29, 1035-1041. doi: 10.1136/gut.29.8.1035

Hirabara, S. M., Silveira, L. R., Alberici, L. C., Leandro, C. V., Lambertucci, R. H., Polimeno, G. C., et al. (2006). Acute effect of fatty acids on metabolism and mitochondrial coupling in skeletal muscle. Biochim. Biophys. Acta 1757, 57-66. doi: 10.1016/j.bbabio.2005.11.007

Hoerr, C. W., Sedgwick, R. S., and Ralston, A. W. (1946). The solubilities of the normal saturated fatty acids. J. Org. Chem. 11, 603-609. doi: 10.1021/ jo0 $1175 \mathrm{a} 025$

James, G. A., Swogger, E., Wolcott, R., Pulcini, E., Secor, P., Sestrich, J., et al. (2008). Biofilms in chronic wounds. Wound Repair. Regen. 16, 37-44. doi: 10.1111/j.1524-475X.2007.00321.x

Jensen, R. G., Ferris, A. M., Lammi-Keefe, C. J., and Henderson, R. A. (1990). Lipids of bovine and human milks: a comparison. J. Dairy Sci. 73, 223-240. doi: 10.3168/jds.S0022-0302(90)78666-3

Kim, B. S., Ott, V., Boecker, A. H., Stromps, J. P., Paul, N. E., Alharbi, Z., et al. (2017). The effect of antiseptics on adipose-derived stem cells. Plast Reconstr. Surg. 139, 625-637. doi: 10.1097/prs.0000000000003125

Kim, S. A., and Rhee, M. S. (2016). Antibacterial activity of caprylic acid for potential application as an active antiseptic ingredient in consumer antiseptics. Int. J. Antimicrob. Agents 48, 765-767. doi: 10.1016/j.ijantimicag.2016. 09.002

Kuhn, D. M., George, T., Chandra, J., Mukherjee, P. K., and Ghannoum, M. A. (2002). Antifungal susceptibility of Candida biofilms: unique efficacy of amphotericin B lipid formulations and echinocandins. Antimicrob. Agents Chemother. 46, 1773-1780. doi: 10.1128/AAC.46.6.1773-1780.2002

\section{AUTHOR CONTRIBUTIONS}

JR, RR, and IR designed the study. RR and NV-C performed planktonic, biofilm, and cytotoxicity assays. JR, RR, NV-C, A-MC, RH, and IR reviewed and analyzed data and contributed conclusions. JR and RR wrote the manuscript. NV-C, A-MC, RH, and IR edited the manuscript. All authors read, commented on, and approved the final manuscript.

Lambers, H., Piessens, S., Bloem, A., Pronk, H., and Finkel, P. (2006). Natural skin surface $\mathrm{pH}$ is on average below 5 , which is beneficial for its resident flora. Int. J. Cosmet. Sci. 28, 359-370. doi: 10.1111/j.1467-2494.2006.00344.x

Lang, W. R. (1955). Vaginal acidity and pH; a review. Obstet. Gynecol. Surv. 10, 546-560. doi: 10.1097/00006254-195508000-00009

Lee, D. S., Sinno, S., and Khachemoune, A. (2011). Honey and wound healing: an overview. Am. J. Clin. Dermatol. 12, 181-190. doi: 10.2165/11538930000000000-00000

Liang, R.-H., Wang, L.-H., Chen, J., Liu, W., and Liu, C.-M. (2015). Alkylated pectin: synthesis, characterization, viscosity and emulsifying properties. Food Hydrocoll. 50, 65-73. doi: 10.1016/j.foodhyd.2015.04.007

Magill, S. S., Edwards, J. R., Bamberg, W., Beldavs, Z. G., Dumyati, G., Kainer, M. A., et al. (2014). Multistate point-prevalence survey of health care-associated infections. N. Engl. J. Med. 370, 1198-1208. doi: 10.1056/NEJMoa1306801

Marina, A. M., Che Man, Y. B., Nazimah, S. A. H., and Amin, I. (2009). Chemical properties of virgin coconut oil. J. Am. Oil Chem. Soc. 86, 301-307. doi: 10.1007/ s11746-009-1351-1

Miquel, S., Lagrafeuille, R., Souweine, B., and Forestier, C. (2016). Anti-biofilm activity as a health issue. Front. Microbiol. 7:592. doi: 10.3389/fmicb.2016.00592

Munarin, F., Tanzi, M. C., and Petrini, P. (2012). Advances in biomedical applications of pectin gels. Int. J. Biol. Macromol. 51, 681-689. doi: 10.1016/j. ijbiomac.2012.07.002

National Center for Emerging and Zoonotic Infectious Diseases, and Division of Foodborne, Waterborne, and Environmental Diseases (2016). Estimates of Foodborne Illness in the United States. Atlanta, GA: Centers For Disease Control and Prevention.

Nobile, C. J., and Johnson, A. D. (2015). Candida albicans biofilms and human disease. Annu. Rev. Microbiol. 69, 71-92. doi: 10.1146/annurev-micro-091014104330

O'Brien, J., Wilson, I., Orton, T., and Pognan, F. (2000). Investigation of the Alamar Blue (resazurin) fluorescent dye for the assessment of mammalian cell cytotoxicity. Eur. J. Biochem. 267, 5421-5426. doi: 10.1046/j.1432-1327.2000. 01606.x

Pieper, B. (2009). Honey-based dressings and wound care: an option for care in the United States. J. Wound Ostomy Continence Nurs. 36, 60-66. doi: 10.1097/01. WON.0000345177.58740.7d

Pieper, B., and Caliri, M. H. (2003). Nontraditional wound care: a review of the evidence for the use of sugar, papaya/papain, and fatty acids. J. Wound Ostomy Continence Nurs. 30, 175-183. doi: 10.1067/mjw.2003.131

Pohl, C., Kock, J., and Thibane, V. (2011). “Antifungal free fatty acids: a Review," in Science against Microbial Pathogens: Communicating Current Research and Technological Advances, ed. A. Mendez-Vilas (Badajoz: Formatex), 61-71.

Rayyan, M., Devlieger, H., Jochum, F., and Allegaert, K. (2012). Short-term use of parenteral nutrition with a lipid emulsion containing a mixture of soybean oil, olive oil, medium-chain triglycerides, and fish oil: a randomized double-blind study in preterm infants. JPEN J. Parenter. Enteral Nutr. 36(Suppl. 1), 81S-94S. doi: 10.1177/0148607111424411

Schreml, S., Szeimies, R. M., Karrer, S., Heinlin, J., Landthaler, M., and Babilas, P. (2010). The impact of the $\mathrm{pH}$ value on skin integrity and cutaneous wound healing. J. Eur. Acad. Dermatol. Venereol. 24, 373-378. doi: 10.1111/j.14683083.2009.03413.x

Sriamornsak, P. (2013). Chemistry of pectin and its pharmaceutical uses: a review. Silpakorn Univ. J. 3, 206-228.

Srivastava, P., and Durgaprasad, S. (2008). Burn wound healing property of Cocos nucifera: an appraisal. Indian J. Pharmacol. 40, 144-146. doi: 10.4103/02537613.43159 
Strober, W. (2015). Trypan blue exclusion test of cell viability. Curr. Protoc. Immunol. 21, A.3B.1-A.3B.2. doi: 10.1002/0471142735.ima03 bs111

Van Acker, H., Van Dijck, P., and Coenye, T. (2014). Molecular mechanisms of antimicrobial tolerance and resistance in bacterial and fungal biofilms. Trends Microbiol. 22, 326-333. doi: 10.1016/j.tim.2014.02.001

Van Houdt, R., and Michiels, C. W. (2010). Biofilm formation and the food industry, a focus on the bacterial outer surface. J. Appl. Microbiol. 109, 11171131. doi: $10.1111 /$ j.1365-2672.2010.04756.x

Wanten, G. J., and Calder, P. C. (2007). Immune modulation by parenteral lipid emulsions. Am. J. Clin. Nutr. 85, 1171-1184.
Conflict of Interest Statement: The authors declare that the research was conducted in the absence of any commercial or financial relationships that could be construed as a potential conflict of interest.

Copyright $\odot 2017$ Rosenblatt, Reitzel, Vargas-Cruz, Chaftari, Hachem and Raad. This is an open-access article distributed under the terms of the Creative Commons Attribution License (CC BY). The use, distribution or reproduction in other forums is permitted, provided the original author(s) or licensor are credited and that the original publication in this journal is cited, in accordance with accepted academic practice. No use, distribution or reproduction is permitted which does not comply with these terms. 